\title{
The role of external gas accretion on galaxy transformations, and evidence of such accretion
}

\author{
Françoise Combes \\ LERMA, CNRS, Observatoire de Paris, 61 Av de l'Observatoire, F-75014 Paris, France \\ email: francoise.combes@obspm.fr
}

\begin{abstract}
Continuously accreting matter from cosmic filaments is one of the main way to assemble mass for galaxies (Keres et al. 2005, Dekel et al. 2009). This external accretion accelerates secular processes, and maintain star formation, but also bar and spiral formation (Bournaud \& Combes 2002), and consequent radial migration. Secular evolution may alleviate the problem of too massive bulge formation in the standard LCDM hierarchical scenario. Inside out formation of galaxies may account for the evolution of the size-mass relation and evolution with redshift. I will show how gas accretion from the inter galactic medium can mimick perturbations due to galaxy interactions (cf Figure 1), and I will describe evidence of such accretion, through warps, polar rings or damped Lyman- $\alpha$ systems.
\end{abstract}

Keywords. hydrodynamics, instabilities, stellar dynamics, turbulence, waves, galaxies: evolution, galaxies: formation

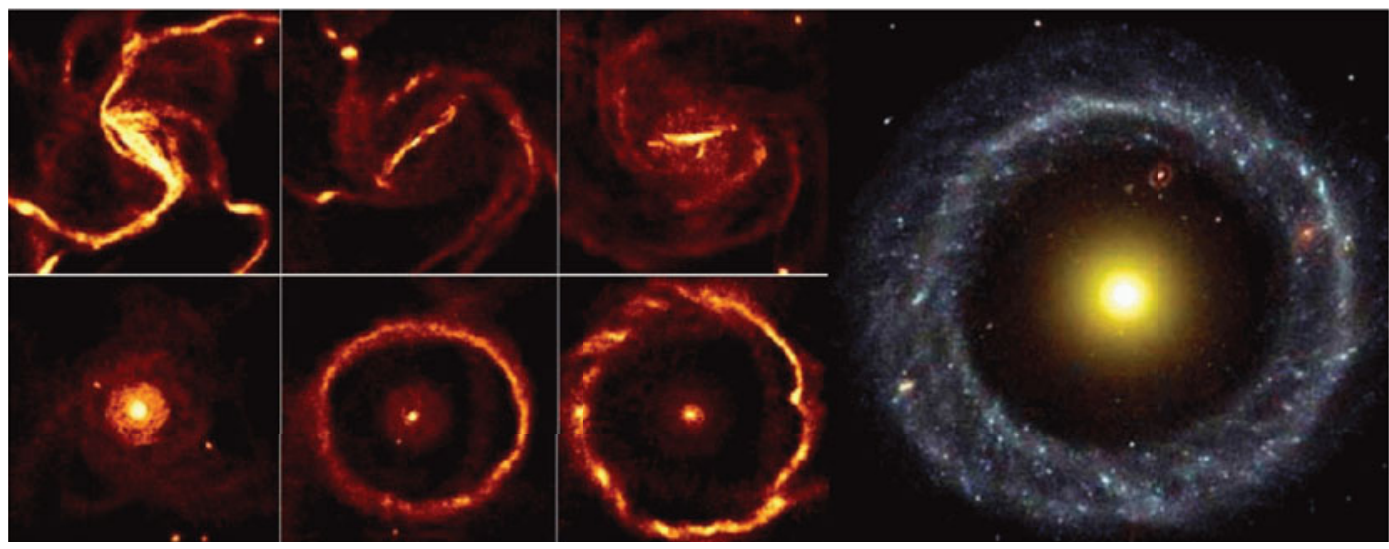

Figure 1. Formation through gas accretion of a ring of gas and new stars around an isolated galaxy (Mastropietro et al.in prep). This could be an explanation for the formation of Hoag-type rings, as shown at right.

\section{References}

Bournaud F. \& Combes F. 2002, A\&A 392, 83

Dekel, A., et al. 2009, Nature 457, 451

Keres, D., et al. 2005, MNRAS 363, 2 